Теорія Ймовір. та Матем. Статист. Вип. 77, 2007
Theor. Probability and Math. Statist.

No. 77, 2008, Pages 147-154

S 0094-9000(09)00753-4

Article electronically published on January 21, 2009

\title{
THE OPTIMAL HEDGING PRICE OF A EUROPEAN TYPE CONTINGENT CLAIM
}

UDC 519.21

\author{
S. V. POSASHKOV
}

\begin{abstract}
A $(B, S)$ financial market is considered in the paper for the case where the volatility is governed by fractional Brownian motion. We prove that the market is incomplete and find the optimal hedging price of a contingent claim that locally minimizes the risk. Under certain assumptions on the price function, we obtain a partial differential equation for the fair hedging price of a contingent claim.
\end{abstract}

\section{INTRODUCTION}

We consider a $(B, S)$ financial market described by the following system of stochastic equations:

$$
\left\{\begin{array}{l}
d S_{t}=a\left(t, S_{t}\right) S_{t} d t+\sigma_{t} S_{t} d W_{t}, \\
d B_{t}=r_{t} B_{t} d t, \quad B_{0}=1,
\end{array} \quad t \in[0, T],\right.
$$

where $W_{t}$ is a standard Brownian motion, $S_{0}$ is a given random variable independent of $W_{t}$, and $r_{t}$ is a nonnegative progressively measurable stochastic process treated as the interest rate.

We assume that the volatility $\sigma_{t}=\rho\left(\tilde{\sigma}_{t}\right)$, where $\rho \in C_{b}^{2}(\mathbb{R})$, is an increasing function and that $\tilde{\sigma}_{t}$ is a stochastic process such that

$$
d \tilde{\sigma}_{t}=\tilde{\alpha}\left(t, \tilde{\sigma}_{t}\right) d t+\tilde{\beta}\left(t, \tilde{\sigma}_{t}\right) d B_{t}^{H}+\varepsilon \tilde{\beta}\left(t, \tilde{\sigma}_{t}\right) d V_{t} .
$$

Here $\varepsilon$ is a positive real number and $B_{t}^{H}$ is fractional Brownian motion with Hurst parameter $H \in(3 / 4,1)$ independent of the process $W_{t}$ (properties of fractional Brownian motion are discussed in the paper [1]). The process $V_{t}$ is a standard Brownian motion independent of the processes $W_{t}$ and $B_{t}^{H}$. We also assume that $\tilde{\sigma}_{0}$ is a given random variable independent of the processes $W_{t}, V_{t}$, and $B_{t}^{H}$.

The classical model with standard Brownian motions is considered, for example, in [2]. It is proved in 3] that the market is incomplete if the volatility is governed by fractional Brownian motion. However, an equation for the optimal hedging price of a contingent claim that locally minimizes the risk is not obtained in [3].

The main feature of the model considered in this paper is the long range dependence of the volatility described by a mixture of standard Brownian motion and fractional Brownian motion with Hurst parameter in the interval $(3 / 4,1)$. We show that one can use the classical technique for this model as well, if $H \in(3 / 4,1)$.

2000 Mathematics Subject Classification. Primary 60H30; Secondary 60J35, 60J65.

Key words and phrases. Optimal hedging price, fractional Brownian motion, European type contingent claim.

(c)2009 American Mathematical Society 
According to the paper [4], the process $M_{t}^{H, \varepsilon}:=\varepsilon V_{t}+B_{t}^{H}$ considered with respect to the flow $\mathcal{F}_{t}^{\prime}, t \in[0, T]$, where

$$
\mathcal{F}_{t}^{\prime}=\sigma\left\{\varepsilon V_{s}+B_{s}^{H}, s \in[0, t]\right\},
$$

is equivalent to standard Brownian motion if $H \in(3 / 4,1)$. Since $S_{0}, \tilde{\sigma}_{0}, W, B^{H}$, and $V$ are independent, the same property holds with respect to the flow $\mathcal{F}_{t}, t \in[0, T]$, where

$$
\mathcal{F}_{t}=\sigma\left\{S_{0}, \tilde{\sigma}_{0}, W_{s}, \varepsilon V_{s}+B_{s}^{H}, s \in[0, t]\right\} .
$$

Applying a Hitsuda result [5], one can represent the process $M_{t}^{H, \varepsilon}$ as follows:

$$
\begin{aligned}
M_{t}^{H, \varepsilon} & =\varepsilon\left(V_{t}+\frac{1}{\varepsilon} B_{t}^{H}\right)=\varepsilon\left(V_{t}^{\prime}+\int_{0}^{t} \int_{0}^{s} r_{\varepsilon}(s, u) d V_{u}^{\prime} d s\right) \\
& =\varepsilon V_{t}^{\prime}+\int_{0}^{t} \int_{0}^{s} \varepsilon r_{\varepsilon}(s, u) d V_{u}^{\prime} d s,
\end{aligned}
$$

where $V^{\prime}$ is another standard Brownian motion adapted to $\mathcal{F}_{t}^{\prime}$ and $\mathcal{F}_{t}$, while $r_{\varepsilon} \in$ $\mathbf{L}^{2}\left([0, T]^{2}\right)$ is a nonrandom kernel that depends on $\varepsilon$ and $H$. An integral equation for $r_{\varepsilon}$ is found in [4]. Note that the long range dependence holds also if $H \in(1 / 2,3 / 4)$. Nevertheless, the process $M_{t}^{H, \varepsilon}$ is not a semimartingale in this case, and thus one cannot use the standard tools of stochastic analysis developed for semimartingales.

Using (1.4), we rewrite (1.2) as follows:

$$
d \tilde{\sigma}_{t}=\tilde{\alpha}_{\omega}\left(\omega, t, \tilde{\sigma}_{t}\right) d t+\tilde{\beta}\left(t, \tilde{\sigma}_{t}\right) d V_{t}^{\prime},
$$

where $\tilde{\alpha}_{\omega}\left(\omega, t, \tilde{\sigma}_{t}\right):=\left(\tilde{\alpha}\left(t, \tilde{\sigma}_{t}\right)+\tilde{\beta}\left(t, \tilde{\sigma}_{t}\right) \int_{0}^{t} \varepsilon r_{\varepsilon}(t, s) d V_{s}^{\prime}\right)$.

The Itô formula implies

$$
d \sigma_{t}=\alpha_{\omega}\left(\omega, t, \sigma_{t}\right) \sigma_{t} d t+\beta\left(t, \sigma_{t}\right) \sigma_{t} d V_{t}^{\prime}
$$

where

$$
\alpha_{\omega}(\omega, t, \sigma):=\left(\rho^{\prime}\left(\rho^{-1}(\sigma)\right) \tilde{\alpha}_{\omega}\left(\omega, t, \rho^{-1}(\sigma)\right)+\frac{\rho^{\prime \prime}\left(\rho^{-1}(\sigma)\right)}{2} \tilde{\beta}\left(t, \rho^{-1}(\sigma)\right)^{2}\right) / \sigma
$$

and

$$
\beta(t, \sigma):=\left(\rho^{\prime}\left(\rho^{-1}(\sigma)\right) \tilde{\beta}\left(t, \rho^{-1}(\sigma)\right)\right) / \sigma .
$$

It is well known that the conditional distribution of $\log S_{t}$ (with respect to the equivalent martingale measure) given $\left\{\sigma_{t}: t \in[0, T]\right\}$, is Gaussian if the interest rate process $r_{t}$ is constant. Then the price of an option can be evaluated by taking the expectation in the Black-Scholes formula. We consider a general interest rate process and a general contingent claim.

We study the question of whether or not the market described by the system of equations (1.1)-(1.2) is incomplete in Section 2.1.

The optimal hedging price for a European contingent claim minimizing a certain risk is obtained in Section 2.2 .

A partial differential equation for the optimal price of a given contingent claim is derived in Section 2.3 .

\section{MAIN PART}

2.1. Incompleteness of the market. First we impose some restrictions on the model and its coefficients. 
Conditions (B):

(B1) There are constants $\sigma_{m}, \sigma^{M} \in(0, \infty)$ such that

$$
\sigma_{m} \leq \rho(x) \leq \sigma^{M} \text { for all } x \in \mathbb{R} .
$$

(B2) $a(t, x)$ is a bounded function.

(B3) $r_{t}$ is a bounded process.

(B4) $\tilde{\alpha}(t, x)$ and $\tilde{\beta}(t, x)$ are bounded functions and there exists a constant $l^{m}$ such that

$$
\tilde{\beta}(t, x) \geq l^{m}>0 \quad \text { for all } t \in[0, T], x \in \mathbb{R} .
$$

Conditions $(C)$ : For all $M>0$, there exists a constant $K_{M}>0$ such that for all $t \geq 0$, $|x| \leq M$, and $|y| \leq M$,

(C1) $|a(t, x)-a(t, y)| \leq K_{M}|x-y|$;

(C2) $|\tilde{\alpha}(t, x)-\tilde{\alpha}(t, y)| \leq K_{M}|x-y|$ and $|\tilde{\beta}(t, x)-\tilde{\beta}(t, y)| \leq K_{M}|x-y|$.

Theorem 2.1. 1. If conditions (B4) and (C2) hold, then equation (1.2) has a unique solution.

2. If conditions (B2) and (C1) hold, then equation (1.1) has a unique solution.

Proof. The first statement of the theorem follows from results of the paper [6]. Indeed, the coefficients $\tilde{\alpha}$ and $\tilde{\beta}$ are bounded (condition (B4)), whence we obtain that they have a linear growth. Moreover, condition (C2) is equivalent to the second condition of Theorem 2.6 in [6. Hence Theorem 2.6 implies the existence and uniqueness of a solution of the stochastic differential equation of type (1.2).

To prove the second statement of the theorem we put $\tilde{S}_{t}=\ln S_{t}$. Then the first equation of system (1.1) is equivalent to the system

$$
d \tilde{S}_{t}=\left(\tilde{a}\left(t, \tilde{S}_{t}\right)-\frac{1}{2} \sigma_{t}^{2}\right) d t+\sigma_{t} d W_{t}
$$

, where $\tilde{a}\left(t, \tilde{S}_{t}\right)=a\left(t, e^{\tilde{S}_{t}}\right)$. The coefficients of (2.1) are bounded and have the Lipshitz property under conditions (B2) and (C1). Thus, system (2.1), resp. (1.1), has a unique solution.

We use a method proposed in 2 to study the incompleteness of the market. Following this method, we consider the discounted capital process $Z_{t}=S_{t} / B_{t}$. Then

$$
d Z_{t}=\frac{S_{t}}{B_{t}}\left(a\left(t, S_{t}\right)-r_{t}\right) d t+\frac{\sigma_{t} S_{t}}{B_{t}} d W_{t} .
$$

Let

where

$$
\eta_{t}=\exp \left(\int_{0}^{t} \gamma_{u} d W_{u}-\frac{1}{2} \int_{0}^{t}\left|\gamma_{u}\right|^{2} d u\right),
$$

$$
\gamma_{t}=\frac{r_{t}-a\left(t, S_{t}\right)}{\sigma_{t}} .
$$

Define the probability measure $\mathrm{P}^{\prime}$ by

$$
\frac{d \mathrm{P}^{\prime}}{d \mathrm{P}}=\eta_{T} \quad \mathrm{P} \text {-a.s. }
$$

Girsanov's theorem implies that the process

$$
W_{t}^{\prime}:=W_{t}-\int_{0}^{t} \gamma_{s} d s
$$


is a $\mathrm{P}^{\prime}$-standard Brownian motion. This means that

$$
Z_{t}=Z_{0}+\int_{0}^{t} \frac{\sigma_{s} S_{s}}{B_{s}} d W_{s}^{\prime}
$$

is a $\mathrm{P}^{\prime}$-martingale.

Theorem 2.2. Let a $(B, S)$ financial market be described by system (1.1). Then the market is incomplete.

Proof. We follow the idea of the proof of Proposition 13.2 in [2]. Let $\eta_{t}^{\prime}:=\exp \left(V_{t}^{\prime}-t / 2\right)$. Conditions (B) imply that $\eta_{t}$ and $\eta_{t}^{\prime}$ are $\mathrm{P}^{\prime}$-square integrable martingales. Consider the measure $\tilde{P}$ defined by

$$
\frac{d \tilde{\mathrm{P}}}{d \mathrm{P}}:=\eta_{t}^{\prime} \eta_{t} .
$$

Then the process $Z_{t}$ is a martingale with respect to the measure $\mathrm{P}^{\prime}$. Thus there are at least two martingale measures. Therefore the market is incomplete.

2.2. Fair price of a contingent claim. Now we consider the optimal price of a European contingent claim $X$ exercised at a moment $T$. If $X$ is attainable, then its price at the moment $t$ is given by

$$
v_{t}=B_{t} \mathrm{E}^{\mathrm{P}^{*}}\left(X B_{T}^{-1} \mid \mathcal{F}_{t}\right)
$$

with respect to any equivalent martingale measure $\mathrm{P}^{*}$. Here $\left\{\mathcal{F}_{t}, t \in[0, T]\right\}$ is a filtration defined on the probability space $\{\Omega, \mathcal{F}, \mathrm{P}\}$ by relation (1.3).

Otherwise, it is natural to use hedge strategies minimizing certain risks if $X$ is not attainable. For example, Föllmer and Schweizer [7] and Schweizer [8 propose to use strategies that minimize the local risk.

We recall some notions of financial mathematics needed in what follows. Let the discounted price process of an asset $X=X_{t}, t \in[0, T]$, be a right continuous semimartingale, and let $X$ possess an expansion with respect to the filtration $\mathcal{F}_{t}, t \in[0, T]$ :

$$
X=X_{0}+M+A,
$$

where $M=M_{t}, t \in[0, T]$, is a square integrable martingale, $M_{0}=0$, whose characteristic $\langle M\rangle_{t}, t \in[0, T]$, is increasing.

The process $A=A_{t}, t \in[0, T]$, in the above representation is a predictable process with bounded variation $|A|$ and such that $A_{0}=0$.

Definition 2.3. A pair of processes $\xi=\xi_{t}, t \in[0, T]$, and $\mu=\mu_{t}, t \in[0, T]$, is called a strategy $\phi$ if

(1) $\xi$ is a predictable process;

(2) $\mathrm{E}\left[\int_{0}^{T} \xi_{s}^{2} d\langle M\rangle_{s}+\left(\int_{0}^{T}\left|\xi_{s}\right| d|A|_{s}\right)^{2}\right]<\infty$;

(3) $\mu$ is an adapted process;

(4) the process $V_{t}(\phi):=\phi_{t} * X_{t}=\xi_{t} X_{t}+\mu_{t}$ is right continuous and $V_{t}(\phi) \in \mathfrak{L}^{2}(\mathrm{P})$, $t \in[0, T]$.

Definition 2.4. A random variable

$$
C_{t}(\phi)=V_{t}(\phi)-\int_{0}^{t} \xi_{s} d X_{s}, \quad t \in[0, T],
$$

is called the discounted capital process $C_{t}(\phi)$ under the strategy

$$
\phi=(\xi, \mu) .
$$


Definition 2.5. Let $\phi=(\xi, \mu)$ be a strategy and let $t \in[0, T]$. A strategy $\tilde{\phi}=(\tilde{\xi}, \tilde{\mu})$ is called an admissible continuation of the strategy $\phi$ from $[0, t]$ to $[0, T]$ if

$$
\tilde{\xi}=\xi \quad \text { for } s \leq t, \quad \tilde{\mu}=\mu \text { for } s<t,
$$

and

$$
V_{T}(\phi)=V_{T}(\tilde{\phi}) \quad \mathrm{P} \text {-a.s. }
$$

A strategy $\Delta=(\delta, \varepsilon)$ is called an admissible variation for $\phi$ in the interval $[t, T]$ if $\phi+\Delta$ is an admissible continuation of $\phi$ from $[0, t]$ to $[0, T]$.

Definition 2.6. A strategy $\phi$ is called a strategy that minimizes the risk if

$$
R_{t}(\tilde{\phi}) \geq R_{t}(\phi) \quad \text { P-a.s. }
$$

for each $t \in[0, T]$ and every admissible continuation $\tilde{\phi}$ of $\phi$, where

$$
R_{t}(\phi)=\mathrm{E}\left[\left(C_{T}(\phi)-C_{t}(\phi)\right)^{2} \mid \mathcal{F}_{t}\right], \quad t \in[0, T] .
$$

Definition 2.7. An admissible variation $\Delta=(\delta, \varepsilon)$ is called a small perturbation if $\delta$ is bounded, $\int_{0}^{T}\left|\delta_{s}\right| d\left|A_{s}\right|$ is finite, and $\delta_{T}=\varepsilon_{T}=0$.

If $\Delta$ is a small perturbation and $(s, t]$ is a subinterval of $[0, T]$, then we define a small perturbation $\left.\Delta\right|_{(s, t]}:=\left(\left.\delta\right|_{(s, t]},\left.\varepsilon\right|_{(s, t]}\right)$ by putting

$$
\left.\delta\right|_{(s, t]}(\omega, u):=\delta_{u}(\omega) I_{(s, t]}(u),\left.\quad \varepsilon\right|_{(s, t]}(\omega, u):=\varepsilon_{u}(\omega) I_{(s, t]}(u) .
$$

Definition 2.8. Let $\phi$ be a strategy, $\Delta$ a small perturbation, and $\tau$ a partition of the interval $[0, T]$. The risk ratio is defined by

$$
r^{\tau}[\phi, \Delta](\omega, t)=\sum_{t_{i} \in \tau} \frac{R_{t_{i}}\left(\phi+\left.\Delta\right|_{\left(t_{i+1}, t_{i}\right]}\right)-R_{t_{i}}(\phi)}{\mathrm{E}\left[\langle M\rangle_{t_{i+1}}-\langle M\rangle_{t_{i}} \mid \mathcal{F}_{t_{i}}\right]}(\omega) \cdot I_{\left(t_{i}, t_{i+1}\right)}(t) .
$$

We say that a strategy $\phi$ locally minimizes the risk if

$$
\lim _{n \rightarrow \infty} \inf r^{\tau_{n}}[\phi, \Delta] \geq 0 \quad \mathrm{P}_{M} \text {-a.s. }
$$

for all small perturbations $\Delta$ and all increasing sequences of partitions $\tau_{n}$ such that $\lim _{n \rightarrow \infty}\left|\tau_{n}\right|=0$. Here $\mathrm{P}_{M}$ is defined as a measure $\mathrm{P} \times\langle M\rangle$ in the space $\tilde{\Omega}=\Omega \times[0, T]$.

A method is proposed in [7] to determine a fair hedging price that locally minimizes the risk for a European type contingent claim. The fair price is determined in [7] with the help of the minimal martingale measure.

Definition 2.9. A martingale measure $\hat{P}$ for $X$ is called the minimal measure for $\mathrm{P}$ if every local $\mathrm{P}$-martingale that is strictly orthogonal to $M$ (with respect to $\mathrm{P}$ ) is a local $\hat{\mathrm{P}}$-martingale.

We show that, similarly to the case where the volatility is not long range dependent, the measure $\mathrm{P}^{\prime}$ is a minimal martingale measure associated with $\mathrm{P}$.

Lemma 2.10. The measure $\mathrm{P}^{\prime}$ defined by equality (2.4) is a minimal martingale measure associated with $\mathrm{P}$.

The proof of this result is the same as that of Lemma 13.3 in [2].

Now we find a formula for the fair price of a hedge that locally minimizes the risk.

Proposition 2.11. Let $X$ be a European type contingent claim with the exercise time $T$ (we do not assume that the contingent claim is attainable). Then the fair price of a hedge that locally minimizes the risk equals

$$
v_{t}=B_{t} \mathrm{E}^{\mathrm{P}^{\prime}}\left(X B_{T}^{-1} \mid \mathcal{F}_{t}\right) .
$$


Proof. Lemma 2.10 implies that $\mathrm{P}^{\prime}$ is a minimal martingale measure. Theorem 3.14 of [7] provides a representation of the fair price of a hedge that minimizes the risk, namely $v_{t}=\mathrm{E}^{\mathrm{P}^{\prime}}(X \mid \mathcal{F})$. To apply Theorem 3.14 of [7], we consider the discounted model (1.1). Then we obtain that the fair price of a hedge that locally minimizes the risk for $X$ is given by (2.5).

2.3. Partial differential equation for the optimal price of a hedge that locally minimizes the risk. In order to derive the differential equation for $v_{t}$, we put

$$
r_{t}=r\left(t, S_{t}\right) \quad \text { and } \quad X=g\left(S_{T}\right),
$$

where $g: \mathbb{R} \rightarrow \mathbb{R}$ is a measurable function. Then

$$
B_{t} B_{T}^{-1}=\exp \left(-\int_{t}^{T} r\left(u, S_{u}\right) d u\right)
$$

Since $\left(S_{t}, \sigma_{t}\right)$ is a Markov process with respect to the flow $\mathcal{F}_{t}$, we have

$$
v_{t}=\mathrm{E}^{\mathrm{P}^{\prime}}\left(g\left(S_{T}\right) \exp \left(-\int_{t}^{T} r\left(u, S_{u}\right) d u\right) \mid\left(S_{t}, \sigma_{t}\right)\right)=: V\left(t, S_{t}, \sigma_{t}\right) .
$$

The process $\left(S_{t}, \sigma_{t}\right)$ considered with respect to the measure $\mathrm{P}^{\prime}$ satisfies the following system of equations:

$$
\left\{\begin{array}{l}
d S_{t}=r\left(t, S_{t}\right) d t+\sigma_{t} S_{t} d W_{t}^{\prime}, \\
d \sigma_{t}=\alpha_{\omega}\left(\omega, t, \sigma_{t}\right) \sigma_{t} d t+\beta\left(t, \sigma_{t}\right) \sigma_{t} d V_{t}^{\prime},
\end{array}\right.
$$

since

$$
\frac{d \mathrm{P}^{\prime}}{d \mathrm{P}}=\exp \left(\int_{0}^{t} \gamma_{u} d W_{u}-\frac{1}{2} \int_{0}^{t}\left|\gamma_{u}\right|^{2} d u\right)
$$

by the definition (2.3) of the function $\gamma_{t}$.

Now we evaluate the generator of the process $\left(S_{t}, \sigma_{t}\right)$ at an arbitrary point $t_{0} \in[0, T]$.

Definition 2.12. The operator

$$
\mathfrak{G}_{t_{0}} f(x):=\lim _{h \downarrow 0} \frac{\mathrm{E}_{x}\left\{f\left(X_{t_{0}+h}\right)\right\}-f(x)}{h},
$$

defined for those functions $f$ for which the limit exists, is called the generator of the Markov process $X_{t}, t \in[0, T]$, at the point $t_{0} \in[0, T)$ given $X_{t_{0}}=x$.

Lemma 2.13. Let all the coefficients of system (2.6) be continuous with respect to all their arguments. Then the generator $\mathfrak{G}_{t_{0}} f(s, \sigma)$ of the process $\left(S_{t}, \sigma_{t}\right)$ at a point $t_{0}$ given $S_{t_{0}}=s$ and $\sigma_{t_{0}}=\sigma$ is as follows:

$$
\begin{aligned}
\mathfrak{G}_{t_{0}} f(s, \sigma)= & \frac{1}{2} \sigma^{2} s^{2} \frac{\partial^{2} f(s, \sigma)}{\partial s^{2}}+\frac{1}{2} \sigma^{2} \beta(t, \sigma)^{2} \frac{\partial^{2} f(s, \sigma)}{\partial \sigma^{2}}+r\left(t_{0}, s\right) s \frac{\partial f(s, \sigma)}{\partial s} \\
& +G\left(t_{0}, s, \sigma\right) \sigma \frac{\partial f(s, \sigma)}{\partial \sigma}
\end{aligned}
$$

for functions $f$ of the class $C_{b}^{2}\left(\mathbb{R}^{2}\right)$, where $G\left(t_{0}, s, \sigma\right):=\mathrm{E}_{s, \sigma}\left\{\alpha_{\omega}\left(\omega, t_{0}, \sigma_{t_{0}}\right)\right\}$. 
Proof. We evaluate the generator explicitly by using Itô's formula:

$$
\begin{aligned}
\mathfrak{G}_{t_{0}} f(s, \sigma)= & \lim _{h \downarrow 0} \frac{\mathrm{E}_{s, \sigma}\left\{f\left(S_{t_{0}+h}, \sigma_{t_{0}+h}\right)\right\}-f(s, \sigma)}{h} \\
= & \lim _{h \downarrow 0} \frac{\mathrm{E}_{s, \sigma}\left\{\int_{0}^{h} \frac{1}{2} \sigma_{t_{0}+u}^{2} S_{t_{0}+u}^{2} \frac{\partial^{2} f\left(S_{t_{0}+u}, \sigma_{t_{0}+u}\right)}{\partial s^{2}} d u\right\}}{h} \\
& +\lim _{h \downarrow 0} \frac{\mathrm{E}_{s, \sigma}\left\{\int_{0}^{h} \sigma_{t_{0}+u}^{2} \beta\left(t_{0}+u, \sigma_{t_{0}+u}\right)^{2} \frac{\partial^{2} f\left(S_{t_{0}+u}, \sigma_{t_{0}+u}\right)}{\partial \sigma^{2}} d u\right\}}{h} \\
& +\lim _{h \downarrow 0} \frac{\mathrm{E}_{s, \sigma}\left\{\int_{0}^{h} r\left(t_{0}+u, S_{t_{0}+u}\right) S_{t_{0}+u} \frac{\partial f\left(S_{t_{0}+u}, \sigma_{t_{0}+u}\right)}{\partial s} d u\right\}}{h} \\
& +\lim _{h \downarrow 0} \frac{\mathrm{E}_{s, \sigma}\left\{\int_{0}^{h} \alpha\left(\omega, t_{0}+u, \sigma_{t_{0}+u}\right) \sigma_{t_{0}+u} \frac{\partial f\left(S_{t_{0}+u}, \sigma_{t_{0}+u}\right)}{\partial \sigma} d u\right\}}{h} \\
= & \frac{1}{2} \sigma^{2} s^{2} \frac{\partial^{2} f(s, \sigma)}{\partial s^{2}}+\frac{1}{2} \sigma^{2} \beta(t, \sigma)^{2} \frac{\partial^{2} f(s, \sigma)}{\partial \sigma^{2}}+r\left(t_{0}, s\right) s \frac{\partial f(s, \sigma)}{\partial s} \\
& +G\left(t_{0}, s, \sigma\right) \sigma \frac{\partial f(s, \sigma)}{\partial \sigma} .
\end{aligned}
$$

Here, one can pass to the limit under the integral sign, due to the assumptions of the lemma, and under the ecpectation sign, due to Lebesgue's dominated convergence theorem, which can be applied here in view of conditions (B) and (C) and the appropriate choice of the class of functions $f$.

We obtain from (2.1) that

$$
d \tilde{S}_{t}=\tilde{a}\left(t, \tilde{S}_{t}, \tilde{\sigma}_{t}\right) d t+\rho\left(\tilde{\sigma}_{t}\right) d W_{t},
$$

where $\tilde{a}\left(t, \tilde{S}_{t}, \tilde{\sigma}_{t}\right)=a\left(t, e^{\tilde{S}_{t}}\right)-\frac{1}{2} \rho\left(\tilde{\sigma}_{t}\right)^{2}$.

Note that $\left(\tilde{S}_{t}, \tilde{\sigma}_{t}\right)$ is a Markov process also with respect to the flow $\mathcal{F}$. Its generator can be evaluated in a similar way, whence we get

$$
\begin{aligned}
\tilde{\mathfrak{G}}_{t} f(\tilde{s}, \tilde{\sigma})= & \frac{1}{2} \rho(\tilde{\sigma})^{2} \frac{\partial^{2} f(\tilde{s}, \tilde{\sigma})}{\partial \tilde{s}^{2}}+\frac{1}{2} \tilde{\beta}(t, \tilde{\sigma})^{2} \frac{\partial^{2} f(\tilde{s}, \tilde{\sigma})}{\partial \tilde{\sigma}^{2}}+\tilde{a}\left(t, \tilde{S}_{t}, \tilde{\sigma}_{t}\right) \frac{\partial f(\tilde{s}, \tilde{\sigma})}{\partial \tilde{s}} \\
& +\tilde{G}(t, \tilde{s}, \tilde{\sigma}) \frac{\partial f(\tilde{s}, \tilde{\sigma})}{\partial \tilde{\sigma}},
\end{aligned}
$$

where $\tilde{G}(t, \tilde{s}, \tilde{\sigma}):=\mathrm{E}_{\tilde{s}, \tilde{\sigma}}\left\{\tilde{\alpha}_{\omega}\left(\omega, t, \tilde{\sigma}_{t}\right)\right\}$. It is clear that

$$
\tilde{V}\left(t, \tilde{S}_{t}, \tilde{\sigma}_{t}\right) \equiv V\left(t, S_{t}, \sigma_{t}\right)=\mathrm{E}^{\mathrm{P}^{\prime}}\left(g\left(e^{\tilde{S}_{T}}\right) \exp \left(-\int_{t}^{T} r\left(u, e^{\tilde{S}_{u}}\right) d u\right) \mid \tilde{S}_{t}, \tilde{\sigma}_{t}\right) .
$$

Put $\tilde{r}(u, \tilde{S}):=r\left(u, e^{\tilde{S}}\right)$.

\section{Theorem 2.14. Let}

(D1) $\tilde{\alpha}, \tilde{\beta}, a, r$, and $\tilde{G}$ be uniformly Hölder continuous; that is, each of these functions has its own positive Hölder exponent, which is constant in the whole domain.

(D2) The rate of growth of $g$ be at most polynomial.

We denote the set of assumptions (D1)-(D2) by (D). Then $V(t, s, \sigma)$ is a unique solution of the partial differential equation

$$
\frac{\partial V}{\partial t}+\frac{1}{2} \sigma^{2} s^{2} \frac{\partial^{2} V}{\partial s^{2}}+\frac{1}{2} \sigma^{2} \beta(t, \sigma)^{2} \frac{\partial^{2} V}{\partial \sigma^{2}}+r(t, s) s \frac{\partial V}{\partial s}+G(t, s, \sigma) \frac{\partial V}{\partial \sigma}=r(t, s) V
$$

with the boundary condition $V(T, s, \sigma)=g(s)$. 
Proof. It follows from Remark 5.7.8 of [9] that the Cauchy problem

$$
-\frac{\partial \tilde{V}}{\partial t}+\tilde{r} \tilde{V}=\tilde{G}_{t} \tilde{V}, \quad \tilde{V}(T, \tilde{s}, \tilde{\sigma})=g\left(e^{\tilde{s}}\right)
$$

has a unique solution satisfying the condition of exponential growth, namely

$$
\max _{t \in[0, T]}|\tilde{V}(t, \tilde{s}, \tilde{\sigma})| \leq M e^{\nu\left(\tilde{s}^{2}+\tilde{\sigma}^{2}\right)} .
$$

Assumptions (B4) and (D) imply all the assumptions of Theorem 5.7.6 in 9], and thus a solution of the Cauchy problem (2.10) has the following stochastic representation:

$$
\tilde{V}\left(t, \tilde{S}_{t}, \tilde{\sigma}_{t}\right)=\mathrm{E}^{\mathrm{P}^{\prime}}\left(g\left(e^{\tilde{S}_{T}}\right) \exp \left(-\int_{t}^{T} r\left(u, e^{\tilde{S}_{u}}\right) d u\right) \mid \tilde{S}_{t}, \tilde{\sigma}_{t}\right),
$$

which coincides with representation (2.8) for the fair price $v_{t}$ of a hedge that locally minimizes the risk.

Theorem 2.14 follows after the change of variables $\tilde{T}:(\tilde{\sigma}, \tilde{s}) \rightarrow(\sigma, s)=\left(\rho(\tilde{\sigma}), e^{\tilde{s}}\right)$ in problem (2.10). This change transforms problem (2.10) to equation (2.9) with the corresponding boundary condition. The uniqueness of the solution of equation (2.9) follows, since the mapping $\tilde{T}$ is bijective.

\section{Concluding Remarks}

We proved that the market described by system (1.1) is incomplete, evaluated the fair price of a hedge for a European type contingent claim that locally minimizes the risk as defined in Definition 2.8, and derived an equation for this price by using the stochastic analysis for semimartingales if $H \in(3 / 4,1)$ and the set of conditions (D) holds.

\section{BIBLIOGRAPHY}

1. I. Norros, E. Valkeila, and J. Virtamo, An elementary approach to a Girsanov formula and other analytical results on fractional Brownian motion, Bernoulli 5(4) (1999), 571-587. MR1704556 (2000f:60053)

2. G. Kallianpur and R. L. Karandinkar, Introduction to Option Pricing Theory, Birkhäuser, Boston, 2000. MR1718056 (2000k:91054)

3. S. V. Posashkov, Studies of the $(B, S)$ market of assets with a stochastic volatility governed by a fractional Brownian motion, Visnyk Kyiv University 2 (2005), 56-61. (Ukrainian)

4. P. Cheridito, Regularizing Fractional Brownian Motion with a View towards Stock Price Modeling, Ph.D. Thesis, Swiss Federal Institute of Technology, Zurich, 2001.

5. M. Hitsuda, Representation of Gaussian processes equivalent to Wiener process, Osaka J. Math. 5 (1968), 299-312. MR0243614(39:4935)

6. Yu. S. Mishura and S. V. Posashkov, Existence and uniqueness of the solution of a stochastic differential equation driven by fractional Brownian motion with a stabilizing term, Teor. Imovir. Matem. Statyst. 76 (2007), 117-124; English transl. in Theory Probab. Math. Statist. 76 (2008), 131-139. MR2368745 (2008j:60143)

7. H. Föllmer and M. Schweizer, Hedging of contingent claim under incomplete information, Applied Stochastic Analysis (M. H. A. Davis and R. J. Elliot, eds.), Gordon and Breach, LondonNew York, 1991, pp. 389-414. MR1108430 (92g:90029)

8. M. Schweizer, Option hedging for semimartingales, Stoch. Process. Appl. 37 (1991), 339-363. MR:1102880 (92c:90025)

9. I. Karatzas and S. E. Shreve, Brownian Motion and Stochastic Calculus, Springer-Verlag, New York, 1991. MR1121940 (92h:60127)

Department of Probability Theory and Mathematical Statistics, Faculty for Mechanics and Mathematics, National Taras Shevchenko University, Academician Glushkov Avenue 6 , KYIV 03127, UKRAINE

E-mail address: corlagon@univ.kiev.ua

Received 31/AUG/2006

Translated by N. SEMENOV 